Bundesgesundheitsbl 2013 56 :885-893

DOI 10.1007/s00103-013-1694-5

Online publiziert: 27. Mai 2013

(c) Springer-Verlag Berlin Heidelberg 2013

\section{Additional material online}

An English full-text version of this article is available at SpringerLink under supplementary material:

dx.doi.org/10.1007/s00103-013-1694-5
J.D. Finger ${ }^{1}$ A. Gößwald ${ }^{1}$ S. Härtel ${ }^{2}$. S. Müters ${ }^{1}$ S. Krug · H. Hölling ${ }^{1}$.

\section{R. Kuhnert ${ }^{1} \cdot$ K. Bö̈ ${ }^{2}$}

${ }^{1}$ Abteilung für Epidemiologie und Gesundheitsmonitoring, Robert Koch-Institut, Berlin

${ }^{2}$ Karlsruher Institut für Technologie (KIT), Institut für Sport und Sportwissenschaft, Karlsruhe

\title{
Messung der kardiorespiratorischen Fitness in der Studie zur Gesundheit Erwachsener in Deutschland (DEGS1)
}

Körperliche Aktivität gilt als wichtiger Faktor zur Vorbeugung und Behandlung chronischer Erkrankungen, zur Steigerung des Wohlbefindens sowie zur Senkung vorzeitiger Sterblichkeit [1, 2]. Körperliche Fitness steht in enger Beziehung zu körperlicher Aktivität und wird durch regelmäßige körperlich-sportliche Betätigung beeinflusst [3]. Körperliche Fitness umfasst gesundheitsbezogene Komponenten wie kardiorespiratorische Fitness (Ausdauer), Kraftausdauer, Muskelkraft, Körperzusammensetzung und Beweglichkeit [4]. Studien berichten, dass die Dosis-Wirkungs-Beziehung zwischen kardiorespiratorischer (aerober) Fitness und Gesundheit sogar noch stärker ausgeprägt zu sein scheint als zwischen körperlicher Aktivität und Gesundheit $[3,5]$. Nach den Ergebnissen der „Aerobics Centre Longitudinal Study" (ACLS) weisen die fittesten Männer und Frauen im obersten Quintil, verglichen mit den am wenigsten fitten Männern und Frauen im untersten Quintil, ein um $43 \%$ bzw. 53\% niedrigeres relatives Risiko für Gesamtsterblichkeit und ein um 47\% bzw. 70\% niedrigeres Risiko für kardiovaskuläre Sterblichkeit auf $[6,7]$. Die kardiorespiratorische Fitness kann durch mäßig anstrengende bis sehr anstrengende aerobe (ausdauerorientierte) Aktivität verbessert werden [8], welche in den aktualisierten Richtlinien zur körperlichen Aktivität der Weltgesundheitsorganisation (WHO) [9] erstmals explizit empfohlen wird. Es ist daher Aufgabe der Prävention und Ge- sundheitsförderung, durch die Förderung aerober körperlich-sportlicher Aktivität das Niveau der kardiorespiratorischen Fitness in der Bevölkerung zu verbessern. Um den Erfolg der entsprechenden Maßnahmen zu überprüfen, besteht ein Forschungsbedarf, die kardiorespiratorische Fitness auf Bevölkerungsebene zu untersuchen und deren Entwicklungstrends zu verfolgen. Verglichen mit subjektiven Befragungsinstrumenten bietet die objektive Messung der kardiorespiratorischen Fitness Vorteile hinsichtlich der Validität und Reliabilität der Messergebnisse [10]. Deshalb wurde in der ersten Erhebungswelle der bundesweiten „Studie zur Gesundheit Erwachsener in Deutschland" (DEGS1) ein submaximaler Fahrradergometertest eingesetzt, um eine objektive Einschätzung der kardiorespiratorischen Fitness Erwachsener im Alter von 18 bis 64 Jahren vorzunehmen [11].

Zielstellung dieses Beitrags ist es, die Messmethodik zu beschreiben, die $\mathrm{Zu}$ sammensetzung der Studienteilnehmer hinsichtlich ihrer Testtauglichkeit darzustellen sowie die Generalisierbarkeit der Messergebnisse einzuschätzen.

\section{Methodik}

\section{Studiendesign}

Die „Studie zur Gesundheit Erwachsener in Deutschland“ (DEGS) ist Bestandteil des Gesundheitsmonitorings des Robert Koch-Instituts (RKI). Konzept und
Design von DEGS sind an anderer Stelle ausführlich beschrieben $[12,13,14,15,16]$. Die erste Erhebungswelle (DEGS1) wurde von 2008 bis 2011 durchgeführt und umfasste Befragungen, Untersuchungen und Tests [17, 18]. Zielpopulation war die in Deutschland lebende Bevölkerung im Alter von 18 bis 79 Jahren. DEGS1 hat ein Mischdesign, das gleichzeitig querund längsschnittliche Analysen ermöglicht. Hierbei wurde eine Einwohnermeldeamtsstichprobe durch ehemalige Teilnehmerinnen und Teilnehmer des Bundes-Gesundheitssurveys 1998 (BGS98) ergänzt. Insgesamt nahmen 8152 Personen teil, darunter 4193 Ersteingeladene (Response 42\%) und 3959 ehemalige Teilnehmerinnen und Teilnehmer des BGS98 (Response 62\%). 7238 Personen besuchten eines der 180 Untersuchungszentren, 914 wurden ausschließlich befragt. Die Nettostichprobe [13] ermöglicht für den Altersbereich von 18 bis 79 Jahren repräsentative Querschnittanalysen und Trendaussagen im Vergleich mit dem BGS98 (n=7988, davon 7116 in Untersuchungszentren). Die Daten der erneut Teilnehmenden sind für Längsschnittanalysen nutzbar. Die Querschnitt- und Trendanalysen werden mit einem Gewichtungsfaktor durchgeführt, der Abweichungen der Stichprobe von der Bevölkerungsstruktur (Stand 31.12.2010) hinsichtlich Alter, Geschlecht, Region und Staatsangehörigkeit sowie Gemeindetyp und Bildung korrigiert [13]. Für den Untersuchungsteil wurde ein gesonderter Gewichtungsfaktor er- 
1. Hat Ihnen jemals ein Arzt gesagt, Sie hätten „etwas am Herzen", und Ihnen nur unter medizinischer Kontrolle Bewegung und Sport empfohlen?

2. Hatten Sie im letzten Monat Schmerzen in der Brust in Ruhe oder bei körperlicher Belastung?

3. Haben Sie Probleme mit der Atmung in Ruhe oder bei körperlicher Belastung?

4. Sind Sie jemals wegen Schwindel gestürzt oder haben Sie schon jemals das Bewusstsein verloren?.

5. Haben Sie Knochen-oder Gelenkprobleme, die sich unter körperlicher Belastung verschlechtern könnten?

6. Hat Ihnen jemals ein Arzt ein Medikament gegen hohen Bluthochdruck oder wegen eines Herzproblems oder Atemproblems verschrieben?...

7. Fühlen Sie sich aufgrund einer vorübergehenden Erkrankung, wie z.B. Fieber oder Erkältung, nicht gut?

8. Sind Sie vermutlich oder sicher schwanger?

9. Kennen Sie irgendeinen weiteren Grund, warum Sie nicht körperlich/sportlich aktiv sein sollten?
Nach ärztl. Begutachtung:

keine Kontraindikation

Ja Nein

Ja Nein

Ja Nein

Ja Nein

Ja Nein

Ja Nein

Ja Nein

Ja Nein

Ja Nein

Abb. $1 \Delta$ Deutsche Version des Physical Activity Readiness-Questionnaires (PAR-Q) [34, 35]

stellt. Bei der Berechnung der Gewichtung für die ehemaligen Teilnehmenden des BGS98 wurde die Wiederteilnahmewahrscheinlichkeit, basierend auf einem logistischen Modell, berücksichtigt. Für die Durchführung von Trendanalysen werden die Daten des Bundesgesundheitssurveys 1998 auf den Bevölkerungsstand zum 31.12.2010 altersadjustiert. Eine Nonresponder-Analyse und der Vergleich einzelner erhobener Indikatoren mit Daten der amtlichen Statistik weisen auf eine hohe Repräsentativität der Stichprobe für die Wohnbevölkerung in Deutschland hin [13].

Alle Teilnehmenden wurden über die Studieninhalte und -ziele informiert und unterzeichneten eine schriftliche Einwilligungserklärung. Das Studienprotokoll wurde von der Ethikkommission der Charité - Universitätsmedizin Berlin sowie vom Bundesbeauftragten für Datenschutz und Informationsfreiheit geprüft und als unbedenklich eingestuft.

\section{Messmethode}

\section{Auswahl des Testverfahrens}

Maximale Belastungstests, in denen mittels Spirometrie die maximale Sauerstoffaufnahmefähigkeit $\left(\mathrm{VO}_{2} \mathrm{max}\right)$ gemessen wird, gelten als Referenzverfahren zur Messung der kardiorespiratorischen
Fitness [19]. Auch wenn maximale Belastungstests im Allgemeinen als sicher gelten, kann das Eintreten von HerzKreislauf-Zwischenfällen und Tod während der Belastung nicht gänzlich ausgeschlossen werden, da sich die Wahrscheinlichkeit, einen plötzlichen Herztod $\mathrm{zu}$ erleiden, unter anstrengender körperlicher Belastung erhöht [20, 21]. Je mehr Risikofaktoren wie hohe Blutfettwerte, Bluthochdruck, Rauchen, Diabetes mellitus oder Vorerkrankungen am Herzen bei einer Person vorliegen, desto höher ist das Zwischenfallrisiko [22]. In klinischen Settings kann von einer Risikorate von bis zu 1 per 2500 Tests ausgegangen werden [23, 24]. Maximale Belastungstests setzen daher die Anwesenheit eines qualifizierten Arztes sowie eine apparative Ausstattung mit MehrkanalEKG-Gerät, vollständige Notfallausrüstung und Defibrillator voraus $[19,25]$. Bei submaximalen Belastungstests ist das Zwischenfallrisiko bei Herzpatienten nur etwa halb so groß wie bei maximalen Belastungstests [23, 26]. Da die Anforderungen an Ausstattung und Personal geringer sind [19] und die Testdauer kürzer ist, stellen submaximale Belastungstests eine kostengünstige Alternative zu maximalen Belastungstests dar; bei einer dennoch adäquaten Einschätzung der kardiorespiratorischen Fitness [6]. In mit DEGS1 vergleichbaren natio- nalen Gesundheitsstudien wie dem USamerikanischen „National Health and Nutrition Examination Survey" (NHANES) [27] oder dem australischen "Survey of the Fitness of Australiens" [28] wurden submaximale Belastungstests mittels Laufband respektive Fahrradergometer eingesetzt. Unter der Annahme, dass sich die Beziehung zwischen der Herzfrequenz und der Sauerstoffaufnahme linear verhält, wurde in den genannten Studien die $\mathrm{VO}_{2}$ max auf Basis von Herzfrequenzdaten per Extrapolation geschätzt [27, 28]. Validierungsstudien haben gezeigt, dass die Korrelationen zwischen der geschätzten $\mathrm{VO}_{2}$ max submaximaler Belastungstest und der gemessenen $\mathrm{VO}_{2}$ max maximaler Belastungstests auf dem Fahrradergometer hoch sind: Es wurden Korrelationskoeffizienten von 0,69 bis 0,98 berichtet $[29,30]$. Maximale und submaximale Belastungstests weisen beide eine hohe Reliabilität auf, es wurden Korrelationskoeffizienten größer als 0,9 berichtet [30]. Um interindividuelle Vergleiche bei maximalen Belastungstests hinsichtlich der $\mathrm{VO}_{2}$ max durchzuführen zu können, muss sichergestellt sein, dass alle Testpersonen gleichermaßen maximal belastet werden, was eine Herausforderung darstellt. Submaximale Tests haben den Vorteil, dass man unabhängig von der Motivation des Teilnehmenden ist, dessen individuelle maximale Leistungsgrenze zu erreichen [31]. Fahrradergometer sind generell günstiger, kleiner, erzeugen weniger Lärm und Bewegung am Oberkörper als Laufbänder, allerdings kommt es bei Untrainierten häufiger zu Ermüdungserscheinungen in der Beinmuskulatur [23].

Nach Expertenkonsultationen sowie einer Risiko-Kosten-Nutzen-Abwägung unter den Rahmenbedingungen eines nationalen mobilen Untersuchungssurveys, wurde zur Erfassung der kardiorespiratorischen Fitness in DEGS1 der Einsatz eines submaximalen Fahrradergometertests bei asymptomatischen Personen im Alter von 18 bis 64 Jahren gewählt. Die kardiorespiratorische Fitness wird dabei anhand der belastungsinduzierten Entwicklung der Herzfrequenz sowie der Laktatkonzentration geschätzt. Die Laktatanalyse ist, ebenso wie die Blutgasmessung und die Spiroergometrie, der Herz- 
frequenzanalyse hinsichtlich der Messgütekriterien überlegen, da sie, sofern eine Mindestleistung erbracht wird, unabhängig von der Mitarbeit des Teilnehmenden ist [32]. Im Kontext eines submaximalen Belastungsprotokolls bietet die laktatbasierte Einschätzung der Leistungsfähigkeit an festgelegten Schwellen im Vergleich zur Bestimmung der $\mathrm{VO}_{2}$ max den Vorteil, dass interpersonelle Vergleiche vorgenommen werden können, ohne dass die Testperson maximal belastet [19] bzw. die $\mathrm{VO}_{2}$ max auf Basis von Linearitätsannahmen [33] geschätzt werden muss.

\section{Einschlusskriterien}

Bei bestehenden kardiovaskulären und pulmonalen Erkrankungen erhöht sich das Risiko von Herz-Kreislauf-Zwischenfällen unter körperlicher Belastung $[19,22,23]$. Deshalb wurde mittels einer modifizierten deutschen Fassung des „Physical Activity Readiness-Questionnaire" (PAR-Q) [34, 35] die Testtauglichkeit der 18- bis 64-jährigen Teilnehmenden anhand von 9 Fragen überprüft, um das Zwischenfallrisiko im Rahmen der Studie zu minimieren (• Abb. 1). Die PAR-Q-Version der Deutschen Gesellschaft für Sportmedizin und Prävention (DGSP) [35] wurde modifiziert, indem die immer zu beachtenden Vorgaben zu vorübergehenden Erkrankungen wie Erkältung, Fieber oder Schwangerschaft in den Fragenkatalog integriert wurden. Der PAR-Q sieht vor, dass bei positiv beantworteten PAR-Q-Fragen der Sachverhalt in einem ärztlichen Gespräch überprüft wird. Dadurch konnte teilweise trotz zunächst positiv beantworteter PAR-Q-Fragen Testtauglichkeit attestiert werden. Als absolute Kontraindikationen galten die nach erneuter Nachfrage durch den Arzt positiv beantworteten PAR-Q-Items „Bewegung und Sport nur unter ärztlicher Kontrolle“, „Einnahme von Medikamenten wegen Herz- oder Atemproblemen" und "Schwangerschaft jenseits der 12. Woche“. Einschlusskriterien für den Fahrradergometertest waren die Zugehörigkeit zur Altersgruppe der 18- bis 64-Jährigen, eine vorliegende Einwilligungserklärung des Teilnehmenden sowie die Testtauglichkeit.

Bundesgesundheitsbl 2013 · 56:885-893 DOI 10.1007/s00103-013-1694-5

(c) Springer-Verlag Berlin Heidelberg 2013

\section{J.D. Finger · A. Gößwald · S. Härtel · S. Müters · S. Krug · H. Hölling · R. Kuhnert · K. Bös Messung der kardiorespiratorischen Fitness in der Studie zur Gesundheit Erwachsener in Deutschland (DEGS1)}

\section{Zusammenfassung}

Ein guter Fitnesszustand ist verbunden mit einem guten Gesundheitszustand und einem geringeren Mortalitätsrisiko. Deshalb wurde in der "Studie zur Gesundheit Erwachsener in Deutschland" (DEGS1) die aerobe Fitness mittels submaximaler Fahrradergometrie bei 18- bis 64-jährigen testtauglichen Erwachsenen ermittelt. Die Gesamtstichprobe umfasste 5263 Personen, von denen 3111 nach dem "Physical Activity Readyness-Questionnaire" als testtauglich eingestuft wurden. 3030 Personen absolvierten einen submaximalen Belastungstest nach WHO-Protokoll (25/25/2). Die Teilnahmequote war $57,2 \%$ bezogen auf die Gesamtstichprobe und $97,4 \%$ bei den Testtauglichen. Neben der kontinuierlichen Herzfrequenzerfassung wurde vor Testbeginn und am Ende jeder Belastungsstufe Ka- pillarblut für die Laktatanalyse abgenommen. Der Test endete nach Überschreiten von 85\% der altersbasierten maximalen Herzfrequenz. $11,9 \%$ der Tests wurden vorzeitig abgebrochen, die durchschnittliche Belastungsdauer betrug 10,8 min, und die angestrebte submaximale Belastung der Testperson in der letzten Belastungsstufe wurde mit einem Wert von 15 auf der 20-Punkte-Borg-Skala im Mittel erreicht. Die bundesweiten Daten können für das Gesundheitsmonitoring, die epidemiologische Forschung und zur Erstellung von Referenzwerten genutzt werden.

\section{Schlüsselwörter}

Kardiorespiratorische Fitness .

Fahrradergometrie - Gesundheitssurvey .

Erwachsene $\cdot$ Deutschland

\section{Measurement of cardiorespiratory fitness in the German Health Interview and Examination Survey for Adults (DEGS1)}

\section{Abstract}

A state of good fitness is related to a better health state and a lower mortality risk. In the German Health Interview and Examination Survey for Adults (DEGS1), aerobic fitness was measured among adults between 18 and 64 years old using a submaximal cycle ergometry test. The total sample comprised 5,263 persons, amongst those 3,111 were categorized as being test-qualified according to the Physical Activity Readiness-Questionnaire. There were 3,030 persons who absolved a submaximal exercise test according to the exercise protocol of the WHO (25/25/2). The test-participation rate was $57.2 \%$ in relation to the total sample and 97.4\% among test-qualified persons. Apart from the continuous heart-rate monitoring, capillary blood was taken prior to starting the test and at the end of each workload stage for performing blood lactate analyses. The test ended when $85 \%$ of the age-predicted maximal heart rate was exceeded. In all $11.9 \%$ of the tests were terminated earlier, the mean exercise duration was $10.8 \mathrm{~min}$, and the anticipated submaximal exertion in the highest workload stage was on average achieved with a mean of 15 on the 20-point RPE scale. The nationwide data can now be used for the national health monitoring system, epidemiological research and for the calculation of reference values. An English full-text version of this article is available at SpringerLink as supplemental.

\section{Keywords}

Cardiorespiratory fitness - Bicycle ergometry . Health survey · Adults · Germany

\section{Gerätetechnische Ausrüstung}

Folgende Materialen wurden im Rahmen des Testaufbaus eingesetzt: kalibrierter Fahrradergometer mit integrierter Blutdruckmessvorrichtung und Polar-Herzfrequenzempfänger (Ergosana CE 0124), Blutdruckmanschetten (Ergosana), Herzfrequenzsender (Oregon), Notebook mit Ergometersoftware
(Dr. Schmidt GmbH), Barcode-Scanner, Laktatmessgerät (EKF-Diagnostics, BiosenC_line), durchblutungsfördernde Salbe, Haemostiletten, Kapillaren $(20 \mu \mathrm{l})$, Defibrillator, ärztlicher Notfallkoffer und 20-Punkte-Borg-Skala [36]. Die BorgSkala wird häufig eingesetzt, um zu beurteilen, ob eine angestrebte Belastung des Teilnehmenden während der Ergometrie erreicht wurde. Dabei entspricht 


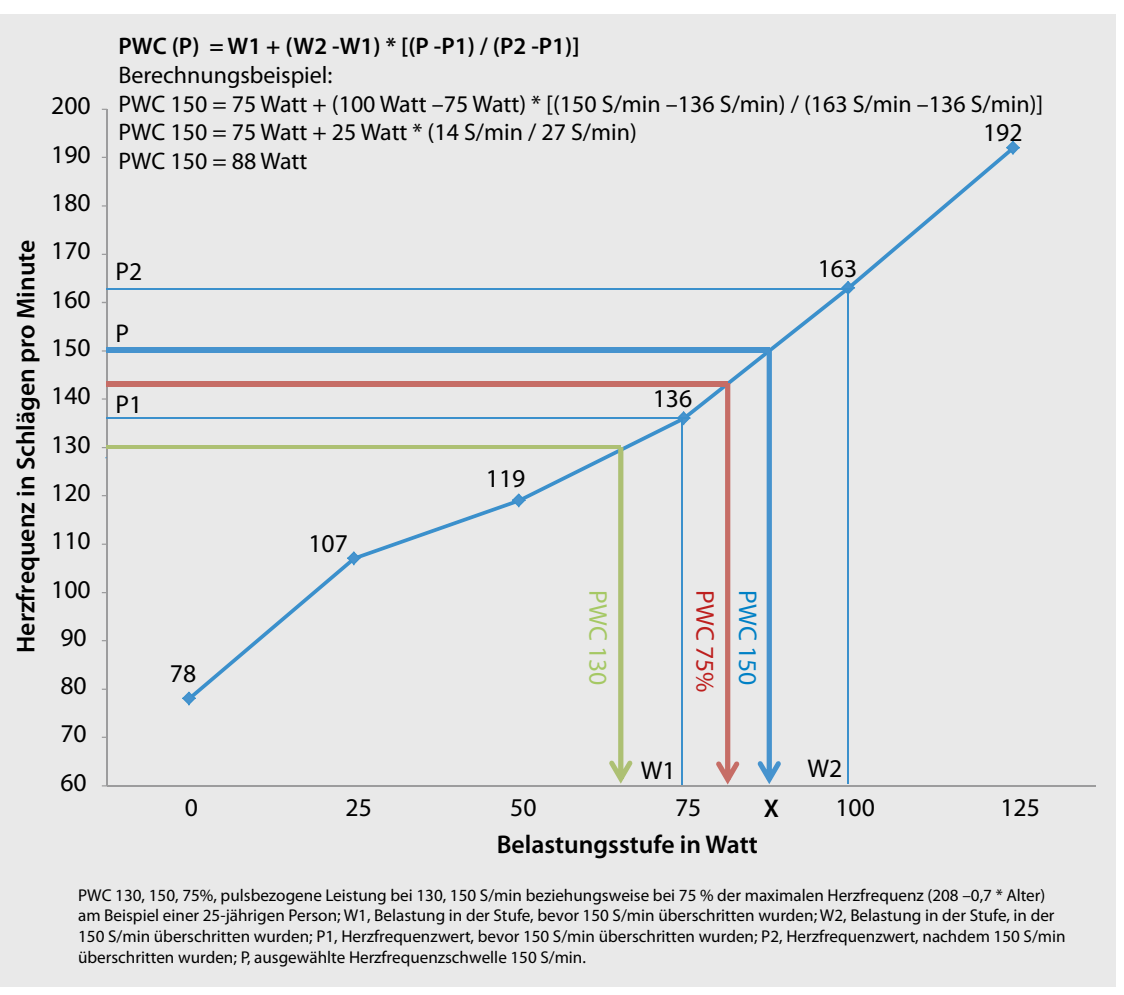

Abb. $2 \Delta$ Bestimmung der Physical Work Capacity (PWC) an fixen und variablen Herzfrequenzschwellen

die individuelle maximale Belastung Borg-Werten von $\geq 17$ [37].

\section{Standardisierter Untersuchungsablauf}

Die Testperson wurde über den Untersuchungsablauf informiert und auf dem Ergometer positioniert. Auf das Ohrläppchen wurde durchblutungsfördernde Salbe aufgebracht und der befeuchtete Brustgurt sowie die Blutdruckmanschette angelegt. Computergestützt wurde die individuelle Zielherzfrequenz bestimmt (maximale Herzfrequenz: 220 minus Lebensalter, davon $85 \%$ [38]). Für die Vorstartlaktatmessung wurden $20 \mu \mathrm{l}$ Kapillarblut aus dem Ohrläppchen abgenommen. Das Software-gesteuerte Testprogramm startete mit der Ermittlung der Vorstartherzfrequenz und des Vorstartblutdrucks. Nach dem Schema der WHO [39] wurde mit $25 \mathrm{~W}$ Belastung begonnen und alle 2 min Software-gesteuert um $25 \mathrm{~W}$ gesteigert. Die Testperson wurde aufgefordert, eine Drehzahl von 70 Umdrehungen pro Minute möglichst konstant zu halten [32, 40]. Die Herzfrequenz wurde kontinuierlich aufgezeich-

\section{Abbruchkriterien}

Es galten folgende Abbruchkriterien [19, 40, 42]: Abnahme der Herzfrequenz trotz steigender Belastung ( $>30$ s), Überschreiten des Blutdrucks von $220 \mathrm{mmHg}$ systolisch bzw. $120 \mathrm{mmHg}$ diastolisch, stark absinkender Blutdruck (mehr als $10 \mathrm{mmHg}$ unterhalb des Anfangsblutdrucks), Herzbeschwerden (Engegefühl), auffällige Blässe, Atemnot, Kopfschmerzen, Schwindel, Sehstörungen, subjektive Erschöpfung, Beinschwäche, Beinkrämpfe, Abfall der Tretkurbelfrequenz auf unter $60 \mathrm{U} / \mathrm{min}$ oder Versagen der Überwachungsgeräte (Blutdruck, Puls).

\section{Auswertungskonzepte}

\section{Herzfrequenzbasierte Auswertung}

Zur Beurteilung der Ausdauerleistungsfähigkeit mittels der Herzfrequenzdaten wird das Konzept der „Physical Work Capacity" (PWC) angewendet [31, 43]. Dem Ansatz zufolge wird die erbrachte Leistung bestimmt, die beim Überschreiten festgelegter Herzfrequenzschwellen bei 130 oder 150 Schlägen pro Minute $(\mathrm{S} / \mathrm{min})$ geleistet wird. Diese wird anschließend zum Körpergewicht der Testperson in Bezug gesetzt. Ab einem Alter von 44 Jahren liegt die Zielherzfrequenz bei submaximalen Belastungstests bereits unter $150 \mathrm{~S} / \mathrm{min}$, demnach kann für diese Altersgruppe methodenbedingt nur noch die $\mathrm{PWC}_{130}$ berechnet werden. Je höher die gewichtsbezogenen PWC-Werte sind, desto besser ist die aerobe Ausdauerleistungsfähigkeit der Teilnehmenden zu bewerten. Da die aufgezeichneten Herzfrequenzwerte in der Regel nicht exakt den für die Zielgruppe relevanten Herzfrequenzschwellen $(130,150 \mathrm{~S} / \mathrm{min})$ entsprechen, werden die $\mathrm{PWC}_{130}$ und $\mathrm{PWC}_{150}$ mithilfe des mathematischen Verfahrens der linearen Interpolation nach der Formel von Rost \& Hollmann [41] bestimmt (• Abb. 2).

Allerdings nimmt die maximale Herzfrequenz mit steigendem Alter kontinuierlich ab [38]. Dieser Umstand wirkt sich bei Altersgruppenvergleichen als Störfaktor aus, wenn die Berechnung pulsbezogener Leistungswerte an fixen Pulsschwellen erfolgt. Eine Herz- 


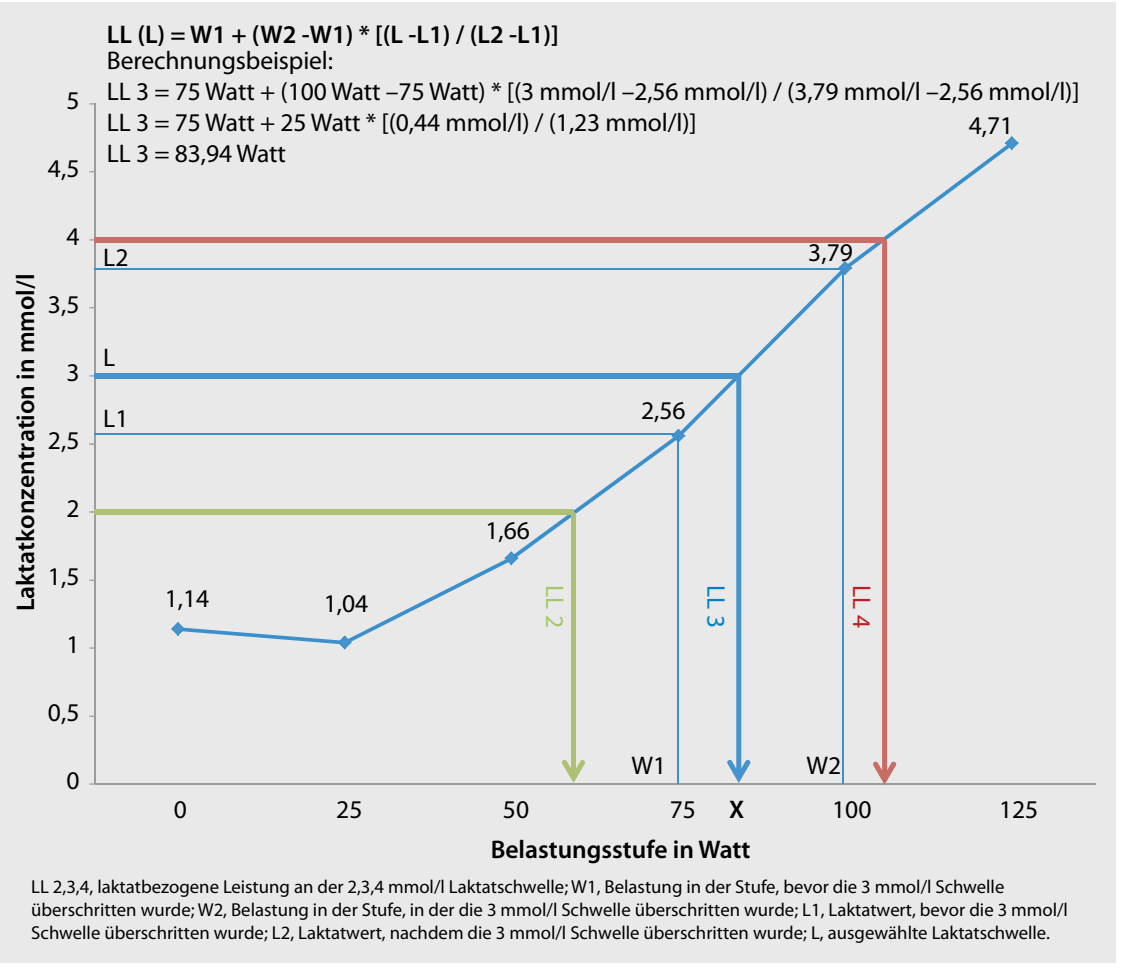

Abb. $3 \Delta$ Bestimmung der laktatbezogenen Leistung (LL) an der 2, 3 und 4 mmol// Laktatschwelle

frequenz von $150 \mathrm{~S} / \mathrm{min}$ entspricht bei einer 20-jährigen Person etwa $75 \%$, bei einer 64-jährigen Person etwa 96\% der maximalen Herzfrequenz. Dies führt zu dem Problem, dass die kardiorespiratorische Fitness jüngerer Personen, verglichen mit der von älteren Personen, im Kontext bevölkerungsbezogener Studien unterschätzt wird [28]. Bei individuellen Schwellenkonzepten hingegen, wird die pulsbezogene Leistung beispielsweise an der variablen Herzfrequenzschwelle von $75 \%$ der altersbasierten maximalen Herzfrequenz ( $\mathrm{PWC}_{75 \%}$ ) [28] bestimmt. Die individuelle Herzfrequenz kann mit der Formel 0,75* $(208-0,7 *$ Alter $)$ [38] bestimmt werden. Anschließend wird die pulsbezogene Leistung $\left(\mathrm{PWC}_{75 \%}\right)$ an der berechneten individuellen Herzfrequenzschwelle mittels grafischer oder rechnerischer Interpolation ermittelt (- Abb. 2). Individuelle Schwellenkonzepte sind im Rahmen von Zusammenhangsanalysen den fixen Schwellenkonzepten vorzuziehen, da für die altersabhängige Abnahme der maximalen Herzfrequenz kontrolliert wird [28].

Zur Auswertung der DEGS1-Daten werden beide Auswertungsverfahren angewendet. Die $\mathrm{PWC}_{130}, 150$ werden

\section{Ergebnisse}

\section{Testausschlüsse, Testabbrüche und Teilnahmequote}

Die Gesamtstichprobe der 18- bis 64-jährigen DEGS1-Teilnehmenden, die untersucht und befragt wurden, belief sich auf 5263 Personen. 316 Personen (6,0\%) wurden als qualitätsneutrale Ausfälle eingestuft, bei denen aus technischen oder organisatorischen Gründen kein Fahrradergometertest durchgeführt werden konnte. 1836 Personen (34,9\%) wurden aufgrund von Testuntauglichkeit nach PAR-Q ausgeschlossen. Mit zunehmendem Alter nahm die Ausschlusswahrscheinlichkeit für den Fahrradergometertest zu. Frauen wurden häufiger vom Test ausgeschlossen als Männer (- Tab. 1). Die „Einnahme von Medikamenten wegen Bluthochdruck, Herzoder Atemproblemen" war die Kontraindikation, die bei den Ausgeschlossenen am häufigsten verzeichnet wurde. Die potenziellen Ausschlussgründe ,jemals wegen Schwindel gestürzt“, „Knochenund Gelenkprobleme“ und „Schmerzen in der Brust “ wurden am häufigsten nach klärenden Rückfragen durch den Arzt als unbedenklich für die Testteilnahme eingestuft (• Tab. 2).

Die endgültige Studienstichprobe für den Fahrradergometertest umfasste 3111 testtaugliche Personen, 1521 Männer und 1590 Frauen. 81 Teilnehmende absolvierten trotz Testtauglichkeit den Ergometertest nicht. Häufigste Ursachen für eine Nichtteilnahme trotz Testtauglichkeit waren Verweigerung $(n=25)$, Verständigungsprobleme $(\mathrm{n}=12)$ und Zeitmangel $(n=8)$ seitens des Teilnehmenden. 3030 Teilnehmende absolvierten den Belastungstest. Bei 359 Teilnehmenden $(11,9 \%)$ wurde der Test vor Erreichen der festgelegten Zielherzfrequenz abgebrochen. Häufigste Gründe für einen vorzeitigen Testabbruch waren subjektive Erschöpfung ( $n=201)$, Überschreitung der Blutdruckgrenzwerte ( $n=93)$, technische Probleme $(n=78)$ sowie Blässe, Atemnot, Schwindel oder Kopfschmerzen $(\mathrm{n}=9)$. Herz-Kreislauf-Zwischenfälle wurden nicht verzeichnet.

Die Testteilnahmequote lag bei $97,4 \%$ bezogen auf die Grundgesamtheit test- 
Tab. 1 Anteil testuntauglicher Personen ${ }^{\mathrm{a}}$ nach dem ärztlichen Gespräch, nach Alter und Geschlecht

\begin{tabular}{|c|c|c|c|c|c|c|c|c|c|c|}
\hline \multirow{2}{*}{$\begin{array}{l}\text { Alters- } \\
\text { gruppe }\end{array}$} & \multicolumn{2}{|c|}{18 bis 29 Jahre } & \multicolumn{2}{|c|}{30 bis 39 Jahre } & \multicolumn{2}{|c|}{40 bis 49 Jahre } & \multicolumn{2}{|c|}{50 bis 64 Jahre } & \multicolumn{2}{|c|}{ Gesamt } \\
\hline & $\mathbf{n}$ & $\%(95 \%-K I)$ & $\mathbf{n}$ & $\%(95 \%-\mathrm{KI})$ & $\mathbf{n}$ & $\%(95 \%-K I)$ & $\mathrm{n}$ & $\%(95 \%-K I)$ & $\mathbf{N}$ & $\%(95 \%-K I)$ \\
\hline \multicolumn{11}{|c|}{ Geschlecht } \\
\hline Frauen & 110 & $20,1(16,8-23,5)$ & 98 & $22,7(18,7-26,7)$ & 227 & $32,6(29,1-31,1)$ & 585 & $53,1(50,1-56,0)$ & 1020 & $36,7(34,9-38,5)$ \\
\hline Männer & 67 & $12,8(9,9-15,6)$ & 76 & $18,5(14,8-22,3)$ & 179 & $29,8(26,2-33,5)$ & 494 & $51,9(48,7-55,1)$ & 816 & $32,8(31,0-34,7)$ \\
\hline Gesamt & 177 & $16,5(14,3-18,8)$ & 174 & $20,7(17,9-23,4)$ & 406 & $31,3(28,8-33,9)$ & 1079 & $52,5(50,4-54,7)$ & 1836 & $34,9(33,6-36,7)$ \\
\hline
\end{tabular}

Tab. 2 Verteilung der Gründe (Mehrfachnennung möglich), die nach dem Physical Activity Readiness-Questionnaire (PAR-Q) zum

Testausschluss führten, stratifiziert nach Geschlecht

\begin{tabular}{|c|c|c|c|c|c|c|c|c|c|c|}
\hline & \multicolumn{4}{|c|}{ Frauen $(n=2610)$} & \multicolumn{4}{|c|}{ Männer (n=2337) } & \multirow{2}{*}{\multicolumn{2}{|c|}{$\begin{array}{l}\text { Testuntauglich nach } \\
\text { Arzt }^{\complement}(n=1836)\end{array}$}} \\
\hline & \multicolumn{2}{|c|}{$\begin{array}{l}\text { Positive } \\
\text { Antworten }^{\mathrm{a}}\end{array}$} & \multicolumn{2}{|c|}{$\begin{array}{l}\text { Rücknahme durch } \\
\text { Arzt }^{\mathrm{b}}\end{array}$} & \multicolumn{2}{|c|}{$\begin{array}{l}\text { Positive } \\
\text { Antworten }^{a}\end{array}$} & \multicolumn{2}{|c|}{$\begin{array}{l}\text { Rücknahme durch } \\
\text { Arzt }^{\text {b }}\end{array}$} & & \\
\hline & $\mathbf{n}$ & $\%$ & $\mathbf{n}$ & $\%$ & $\mathbf{n}$ & $\%$ & n & $\%$ & $\mathbf{n}$ & $\%$ \\
\hline \multicolumn{11}{|l|}{ Ausschlussgründe } \\
\hline Sport nur unter ärztlicher Kontrolle & 216 & 8,3 & 45 & 20,8 & 224 & 9,6 & 45 & 20,1 & 350 & 19,1 \\
\hline Schmerzen in der Brust & 332 & 12,7 & 121 & 36,4 & 253 & 10,8 & 106 & 41,9 & 358 & 19,5 \\
\hline Probleme mit Atmung & 551 & 21,1 & 188 & 34,1 & 342 & 14,6 & 113 & 33,0 & 592 & 32,2 \\
\hline Jemals wegen Schwindel gestürzt & 539 & 20,7 & 301 & 55,8 & 317 & 13,6 & 164 & 51,7 & 391 & 21,3 \\
\hline Knochen- oder Gelenkprobleme & 806 & 30,9 & 316 & 39,2 & 642 & 27,5 & 280 & 43,6 & 852 & 46,4 \\
\hline Medikation $^{d}$ & 731 & 28,0 & 110 & 15,0 & 647 & 27,7 & 91 & 14,1 & 1177 & 64,1 \\
\hline Fieber oder Erkältung & 247 & 9,5 & 53 & 21,5 & 134 & 5,7 & 25 & 18,7 & 303 & 16,5 \\
\hline Vermutlich oder sicher schwanger & 32 & 1,2 & 7 & 21,9 & - & - & - & - & 25 & 1,4 \\
\hline Weitere Kontraindikationen & 147 & 5,6 & 22 & 15,0 & 136 & 5,8 & 17 & 12,5 & 244 & 13,3 \\
\hline
\end{tabular}

tauglicher Personen, 61,2\% bezogen auf die Bruttostichprobe (bereinigt um qualitätsneutrale Ausfälle) und 57,2\% bezogen auf die Gesamtstichprobe. Insgesamt konnte bei 2843 Personen die gewichtsbezogene $\mathrm{PWC}_{130}$ bestimmt werden, bei 2827 die $\mathrm{PWC}_{75 \%}$ und bei 1586 die $\mathrm{PWC}_{150}$ (• Abb. 4).

\section{Testdauer, Anzahl der Belastungsstufen und subjektives Anstrengungsempfinden}

Die durchschnittliche Belastungsdauer betrug 10,8 min. Einschließlich der 5-minütigen Erholungsphase sowie der Testeinweisung nahm der Fahrradergometertest im Untersuchungsablauf etwa 15-20 min ein. Die maximale Anzahl absolvierter Belastungsstufen belief sich auf 12 Stufen $(300 \mathrm{~W})$.

Im Durchschnitt wurden von den Teilnehmenden 5,4 Belastungsstufen absolviert. Dies entspricht einer Leistung von 135 W. Der Mittelwert des subjektiven Anstrengungsempfindens nach der Borg-Skala [36] in der letzten Belastungsstufe entsprach bei Frauen einem Borg-Wert von 14,9 und bei Männern 15,0 , was dem Belastungsempfinden „anstrengend" entspricht.

\section{Diskussion}

\section{Testtauglichkeit und Teilnehmersicherheit}

In DEGS1 wurde eine Testausschlussrate aufgrund von Krankheit und Symptomen nach PAR-Q in Höhe von 34,9\% verzeichnet. Die Ausschlussrate nahm mit steigendem Alter zu. Damit wurde die auf Grundlage der Krankheitsprävalenzen der Gesundheitsberichterstattung für Deutschland (GBE) vor Studienbeginn erwartete Testausschlussrate nach PAR-Q von etwa einem Drittel der Teilnehmenden geringfügig überschritten. Laut der Studie „Gesundheit in Deutsch- land aktuell 2009“ (GEDA09) lag die Prävalenz chronischer Erkrankungen bei den 18 - bis 64 -Jährigen bei etwa $33 \%$, wobei auch hier die Prävalenz mit steigendem Alter zunahm [46]. Auch der Anteil von $28 \%$ der Testpersonen, die angaben, aufgrund von Bluthochdruck bzw. Atemoder Herzproblemen Medikamente einzunehmen, entspricht in etwa der 12-Monats-Prävalenz des Bluthochdrucks in Höhe von etwa 26\% laut GEDA09.

Bei der Konzipierung des Tests wurde besonderer Wert darauf gelegt, eine hohe Datenqualität zu erreichen und gleichzeitig das Risiko der Teilnehmenden für einen Herz-Kreislauf-Zwischenfall so gering wie möglich zu halten. Das mittlere subjektive Anstrengungsempfinden am Ende der letzten Belastungsstufe entsprach in DEGS1 einem Borg-Wert von etwa 15 („,anstrengend“) [36], was - wie durch das Studiendesign angestrebt einer submaximalen Belastung des Teilnehmenden entspricht. Zudem kam es im gesamten Studienverlauf zu keinem 


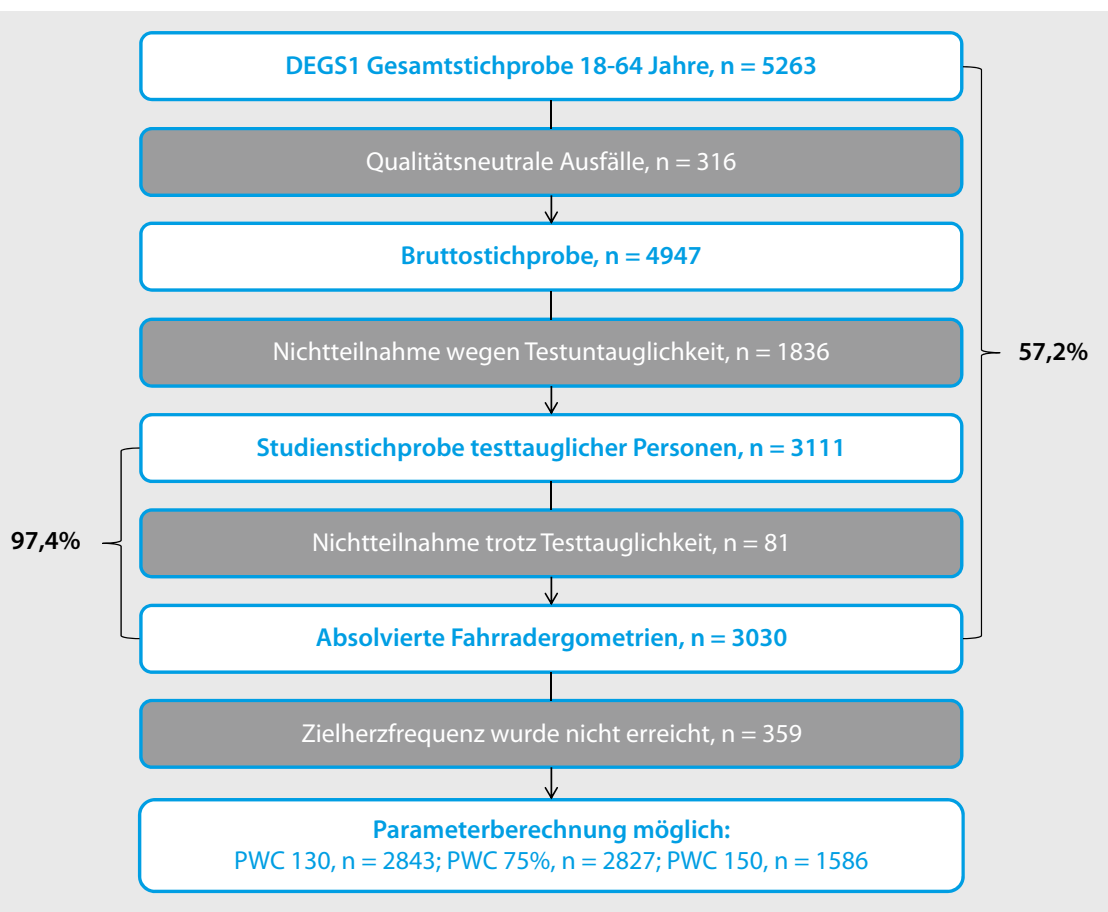

Abb. $4 \Delta$ Bestimmung der Testteilnahmequote bezogen auf die Gesamt- und Studienstichprobe

Herz-Kreislauf-Zwischenfall. Mit einer im Durchschnitt erreichten submaximalen Belastung bei gleichzeitiger Vermeidung von Herz-Kreislauf-Zwischenfällen wurde die angestrebte Balance zwischen ausreichender Belastung und Sicherheit erzielt.

\section{Interne und externe Validität}

Die interne Validität der erhobenen Messparameter ist im Kontext bevölkerungsbezogener Studien, in denen bevorzugt subjektive Befragungsinstrumente zur Erfassung körperlicher Aktivität und Fitness eingesetzt werden, als hoch zu bewerten [3]. Die Entscheidung für ein submaximales Belastungsprotokoll und die Schätzung der kardiorespiratorischen Fitness auf Basis von Herzfrequenzdaten, entspricht dem Vorgehen in vergleichbaren nationalen Gesundheitsstudien [27, 28]. Im Vergleich zur Referenzstandardmessung der $\mathrm{VO}_{2} \max$ in maximalen Belastungstests muss bei der herzfrequenzbasierten Einschätzung der Fitness in submaximalen Belastungstests jedoch von einer reduzierten Validität ausgegangen werden: Es wurden Korrelationskoeffizienten von 0,69 bis 0,98 berichtet $[29,30]$. Herzfrequenzparame- ter gelten, verglichen mit Sauerstoffaufnahme- und Laktatparametern, als weniger valide, da die Herzfrequenz von Lebensalter, Geschlecht, Trainingszustand, Körpertemperatur, Umgebungstemperatur und vegetativen Faktoren abhängig ist [47], welche zu einer erhöhten Schwankungsbreite der Herzfrequenz beitragen. Die zusätzliche Erfassung von Laktatparametern stellt, verglichen mit nationalen Gesundheitsstudien in anderen Ländern, einen Mehrwert der Studie dar, da Laktatparameter gleichermaßen wie die der Sauerstoffaufnahme zur validen Beurteilung der aeroben Leistungsfähigkeit geeignet sind [47].

Die Ergebnisse sind verallgemeinerbar für die Grundgesamtheit nach PAR-Q testtauglicher Erwachsener in der Altersgruppe von 18 bis 64. Die Generalisierbarkeit der Ergebnisse bezogen auf die Gesamtbevölkerung in dieser Altersgruppe ist aufgrund der positiven Selektierung nach PAR-Q testtauglicher Personen eingeschränkt. Es ist zu erwarten, dass testuntaugliche Personen durchschnittlich eine geringere kardiorespiratorische Fitness aufweisen als testtaugliche Personen. Beobachtungsstudien weisen auf einen wechselseitig negativen $\mathrm{Zu}$ sammenhang zwischen kardiorespirato- rischer Fitness und der Prävalenz von Bluthochdruck hin [48]. Da das Vorliegen von Bluthochdruck die Wahrscheinlichkeit erhöht, nach PAR-Q vom Test ausgeschlossen zu werden, kann davon ausgegangen werden, dass die kardiorespiratorische Leistungsfähigkeit in der Gesamtbevölkerung geringer ausgeprägt ist als bei der Grundgesamtheit testtauglicher Personen. Dieser Selektionsbias ist in höheren Altersgruppen stärker ausgeprägt, da mit zunehmendem Alter der Anteil testuntauglicher Personen zunimmt.

\section{Ausblick}

Die derzeit in der Fachöffentlichkeit empfohlenen Richtwerte zur PWC [8, $40,42,47$ ] wurden vor rund 30 Jahren auf Basis einer vergleichsweise kleinen nicht randomisierten Stichprobe (Anzahl der Männer =123; Anzahl der Frauen nicht angegeben) erstellt, die aus untrainierten Erwachsenen bestand, die das Institut der Arbeitsgruppe besuchten [41]. Bundesweite Richtwerte zur laktatbasierten Einschätzung der Leistungsfähigkeit liegen nach unserem Wissen bislang nicht vor [49]. DEGS1 ermöglicht die Erstellung aktueller herzfrequenzund laktatbasierter Richtwerte zur kardiorespiratorischen Fitness auf Basis einer bundesweiten Stichprobe. Es ist geplant, die objektiven Daten zur kardiorespiratorischen Fitness mit den ebenfalls in DEGS1 erhobenen subjektiven Daten zur körperlich-sportlichen Aktivität zu vergleichen sowie Zusammenhangsanalysen zwischen der kardiorespiratorischen Fitness und weiterer in DEGS1 erhobener Variablen durchzuführen. Neben der Untersuchung soziodemografischer Determinanten bietet die Breite der in DEGS1 erhobenen Gesundheitsvariablen [14] vielfältige Möglichkeiten, die Beziehung zwischen kardiorespiratorischer Fitness und Gesundheit zu untersuchen. Darüber hinaus ist geplant, die DEGS1-Teilnehmenden in regelmäßigen Abständen erneut $\mathrm{zu}$ untersuchen und zu befragen. Dadurch bietet sich perspektivisch die Möglichkeit, Trendanalysen und Lebensverlaufsanalysen durchzuführen, sowie kausale Zusammenhänge zwischen kardiorespiratorischer Fit- 
ness und der Entwicklung von Übergewicht und chronischen Erkrankungen im Längsschnitt zu untersuchen.

\section{Fazit}

Aufgrund der zunehmenden Bedeutung aerober körperlicher Aktivität für die Prävention und Gesundheitsförderung, stellt die objektive Erfassung der aeroben Fitness eine sinnvolle Ergänzung des Untersuchungsspektrums des Surveys dar. Die angewendete Messmethode entspricht dem internationalen Standard bevölkerungsbezogener Gesundheitsstudien. Die Testausschlussquote von etwa $35 \%$ lag im Rahmen der Prävalenz der als Kontraindikation definierten Erkrankungen und Medikationen in der untersuchten Altersgruppe. Durch das ärztliche Gespräch konnten Personen, die zunächst nach PAR-Q als testuntauglich eingeschätzt wurden, dennoch zum Test zugelassen werden. Die Validität von Herzfrequenzparametern submaximaler Belastungstests ist geringer verglichen mit den Parametern der Spiroergometrie im Rahmen maximaler Belastungstests. Im Kontext nationaler Gesundheitsstudien, in denen meist subjektive Befragungsinstrumente eingesetzt werden, stellt die herzfrequenzbasierte objektive Messung der Fitness einen Validitätsgewinn dar. Die zusätzliche Erfassung von Laktatparametern stellt einen weiteren Mehrwert der Studie dar, da Laktatparameter zur validen Bestimmung der aeroben Ausdauer geeignet sind. Mit den DEGS1-Fahrradergometerdaten liegen nun bundesweite Daten zur kardiorespiratorischen Fitness testtauglicher Erwachsener im Alter von 18 bis 64 Jahren vor, die für das Gesundheitsmonitoring, die Erforschung epidemiologischer Fragestellungen sowie zur Erstellung von Referenzwerten genutzt werden können.

\section{Korrespondenzadresse}

\section{J.D. Finger}

Abteilung für Epidemiologie und Gesundheitsmonitoring, Robert Koch-Institut General-Pape-Str. 62-66, 12101 Berlin FingerJ@rki.de

Finanzierung der Studie. Die Studie wurde finanziert mit Mitteln des Robert Koch-Instituts und des Bundesministeriums für Gesundheit.

Interessenkonflikt. Der korrespondierende Autor gibt für sich und seine Koautoren an, dass kein Interessenkonflikt besteht.

\section{Literatur}

1. Sallis JF, Owen N (1999) Physical activity and behavioral medicine. Sage, Thousand Oaks

2. National Center for Chronic Disease Prevention and Health Promotion (1999) Physical activity and health: a report of the Surgeon General. United States Department of Health and Human Service, Atlanta

3. Blair SN, Cheng Y, Scott Holder J (2001) Is physical activity or physical fitness more important in defining health benefits? Med Sci Sports Exerc 33:S379-S399

4. Caspersen CJ, Powell KE, Christenson GM (1985) Physical activity, exercise, and physical fitness: definitions and distinctions for health-related research. Public Health Rep 100:126-131

5. Brandes M (2012) Körperliche Aktivität oder Fitness: Was ist wichtiger für die Gesundheit? Bundesgesundheitsbl Gesundheitsforsch Gesundheitsschutz 55:96-101

6. Lee D, Artero EG, Sui X, Blair SN (2010) Review: mortality trends in the general population: the importance of cardiorespiratory fitness. J Psychopharmacol 24:27-35

7. Blair SN, Kohl HW, Barlow CE et al (1995) Changes in physical fitness and all-cause mortality. JAMA 273:1093

8. Hollmann W, Strüder HK (2009) Sportmedizin: Grundlagen von körperlicher Aktivität, Training und Präventivmedizin. Schattauer, Stuttgart

9. World Health Organisation (2010) Global recommendations on physical activity for health. WHO Press, Geneva

10. Shephard RJ (2003) Limits to the measurement of habitual physical activity by questionnaires. $\mathrm{Br} \mathrm{J}$ Sports Med 37:197-206

11. Robert Koch-Institut (2009) Informationen zu DEGS: Fahrradergometrie. Epidemiol Bull 40:399

12. Gößwald $A$, Lange $M$, Kamtsiuris $P$, Kurth $B M$ (2012) DEGS: Studie zur Gesundheit Erwachsener in Deutschland. Bundesweite Quer- und Längsschnittstudie im Rahmen des Gesundheitsmonitorings des Robert Koch-Instituts. Bundesgesundheitsbl Gesundheitsforsch Gesundheits schutz 55:775-780

13. Kamtsiuris $P$, Lange M, Hoffmann R et al (2013) Die erste Welle der Studie zur Gesundheit Erwachsener in Deutschland (DEGS1). Stichprobendesign, Response, Gewichtung und Repräsentativität. Bundesgesundheitsbl Gesundheitsforsch Gesundheitsschutz 56:620-630

14. Kurth BM (2012) Das Gesundheitsmonitoring was es enthält und wie es genutzt werden kann. Public Health Forum 20(76):4.e1-4.e3
15. Kurth BM, Lange C, Kamtsiuris P, Hölling H (2009) Gesundheitsmonitoring am Robert Koch-Institut. Sachstand und Perspektiven. Bundesgesundheitsbl Gesundheitsforsch Gesundheitsschutz 52:557-570

16. Scheidt-Nave C, Kamtsiuris $P$, Gößwald A et al (2012) German Health Interview and Examination Survey for Adults (DEGS) - design, objectives and implementation of the first data collection wave. BMC Public Health 12:730

17. Gößwald A, Lange M, Dölle R, Hölling H (2013) Die erste Welle der Studie zur Gesundheit Erwachsener in Deutschland (DEGS1). Gewinnung von Studienteilnehmenden, Durchführung der Feldarbeit und Qualitätsmanagement. Bundesgesundheitsbl Gesundheitsforsch Gesundheitsschutz 56:611-619

18. Robert Koch-Institut (Hrsg) (2009) DEGS: Studie zur Gesundheit Erwachsener in Deutschland Projektbeschreibung. Beiträge zur Gesundheitsberichterstattung des Bundes. RKI, Berlin

19. American College of Sports Medicine (2006) ACSM's guidelines for exercise testing and prescription. Lippincott Williams \& Wilkins, Philadelphia

20. Thompson PD, Franklin BA, Balady GJ et al (2007) Exercise and acute cardiovascular events placing the risks into perspective: a scientific statement from the American Heart Association Council on Nutrition, Physical Activity, and Metabolism and the Council on Clinical Cardiology. Circulation 115:2358-2368

21. Rognmo O, Moholdt T, Bakken H et al (2012) Cardiovascular risk of high- versus moderate-intensity aerobic exercise in coronary heart disease patients. Circulation 126:1436-1440

22. Fletcher GF, Mills WC, Taylor WC (2006) Update on exercise stress testing. Am Fam Physician 74:1749-1754

23. Gibbons RJ, Balady GJ, Beasley JW et al (1997) ACC/AHA guidelines for exercise testing. A report of the American College of Cardiology/American Heart Association Task Force on Practice Guidelines (Committee on Exercise Testing). J Am Coll Cardiol 30:260

24. Stuart RJ, Ellestad MH (1980) National survey of exercise stress testing facilities. Chest J 77:94-97

25. Deutsche Gesellschaft für Sportmedizin und Prävention (DGSP). Expertenkommission: Boldt F, Berbalk A, Halle M, Hoffmann G, Löllgen $H$, Schmidt Trucksäß A, Urhausen A, Völker K, Zurstegge M (2002) Leitlinien zur Belastungsuntersuchung in der Sportmedizin. Frankfurt am Main

26. Juneau $M$, Colles $P$, Théroux $P$ et al (1992) Symptom-limited versus low level exercise testing before hospital discharge after myocardial infarction. J Am Coll Cardiol 20:927-933

27. Wang CY, Haskell WL, Farrell SW et al (2010) Cardiorespiratory fitness levels among US adults 20 49 years of age: findings from the $1999-2004 \mathrm{Na}-$ tional Health and Nutrition Examination Survey. Am J Epidemiol 171:426-435

28. Gore CJ, Booth ML, Bauman A, Owen N (1999) Utility of pwc $75 \%$ as an estimate of aerobic power in epidemiological and population-based studies. Med Sci Sports Exerc 31:348

29. Noonan V, Dean E (2000) Submaximal exercise testing: clinical application and interpretation. Phys Ther 80:782-807

30. Eng JJ, Dawson AS, Chu KS (2004) Submaximal exercise in persons with stroke: test-retest reliability and concurrent validity with maximal oxygen consumption. Arch Phys Med Rehabil 85:113118 
31. Wahlund $H$ (1948) Determinants of the physical work capacity. A physiological and clinical study with special reference to standardization of cardio-pulmonary functional test. Acta Med Scand 215(Suppl):83-98

32. Löllgen H, Fahrenkrog U, Völker K et al (2010) Allgemeine Aspekte der Ergometrie. In: Löllgen H, Erdmann E, Gitt AK (Hrsg) Ergometrie. Belastungsuntersuchungen in Klinik und Praxis. Springer Medizinverlag, Heidelberg, S 21-60

33. Fitchett M (1985) Predictability of VO2 max from submaximal cycle ergometer and bench stepping tests. Br J Sports Med 19:85-88

34. Canadian Society for Exercise Physiology (2012) Physical activity readiness questionnaire - PAR-Q (revised 2002). http://csep.ca/cmfiles/publications/parq/par-q.pdf (Zugegriffen: 30.11.2012)

35. Deutsche Gesellschaft für Sportmedizin und Prävention (DGSP) (2012) PAR-Q-Fragebogen (deutsche Fassung). http://www.dgsp.de/downloads/ allgemein/Eingangsfragebogen.pdf (Zugegriffen: 30.11.2012)

36. Borg G (2004) Anstrengungsempfinden und körperliche Aktivität. Dtsch Ärztebl 101:A1016A1021
37. Löllgen H, Ulmer H-V (2004) Das „Gespräch“ während der Ergometrie: Die Borg-Skala. Dtsch Ärztebl 101:A1014-A1015

38. Tanaka H, Monahan KD, Seals DR (2001) Age-predicted maximal heart rate revisited. J Am Coll Cardiol 37:153-156

39. Lange Andersen K, Shephard R, Denolin $\mathrm{H}$ et al (1971) Fundamentals of exercise testing. World Health Organisation, Geneva

40. Rost R (Hrsg) (2001) Lehrbuch der Sportmedizin. Deutscher Ärzte-Verlag, Köln

41. Rost R, Hollmann W (1982) Belastungsuntersuchungen in der Praxis. Thieme, Stuttgart

42. De Marées H (2002) Sportphysiologie, 9. überarbeitete und erweiterte Aufl. Sport und Buch Strauss, Köln

43. Astrand I (1958) The physical work capacity of workers 50-64 years old. Acta Physiol Scand 42:73-86

44. Keul J, Simon G, Berg A et al (1979) Bestimmung der individuellen anaeroben Schwelle zur Leistungsbewertung und Trainingsgestaltung. Dtsch Z Sportmed 30:212-218
45. Stegmann H, Kindermann W, Schnabel A (1981) Lactate kinetics and individual anaerobic threshold. Int J Sports Med 2:160-165

46. Robert Koch-Institut (2010) Daten und Fakten: Ergebnisse der Studie "Gesundheit in Deutschland aktuell 2009". Beiträge zur Gesundheitsberichterstattung des Bundes. In: Gesundheitsberichterstattung des Bundes. Robert Koch-Institut, Berlin

47. Hollmann W, Strüder HK, Predel HG, Tagarakis CVM (2006) Teil 3. Untersuchungsparameter und -verfahren. In: Hollmann W, Strüder HK, Predel HG, Tagarakis CVM (Hrsg) Spiroergometrie. Kardiopulmonale Leistungsdiagnostik des Gesunden und Kranken. Schattauer, Stuttgart, S 74-130

48. Carnethon MR, Gulati M, Greenland P (2005) Prevalence and cardiovascular disease correlates of low cardiorespiratory fitness in adolescents and adults. JAMA 294:2981-2988

49. Heck H, Beneke R (2008) 30 Jahre Laktatschwellen - was bleibt zu tun? Dtsch Z Sportmed 59:297

\section{Hier steht eine Anzeige.}

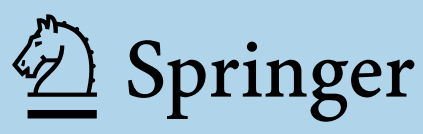

